\title{
BMJ Open Bragatston study protocol: a multicentre cohort study on automated quantification of cardiovascular calcifications on radiotherapy planning CT scans for cardiovascular risk prediction in patients with breast cancer
}

\author{
Marleen J Emaus, ${ }^{1}$ Ivana Išgum, ${ }^{2}$ Sanne G M van Velzen, ${ }^{2}$ \\ H J G Desirée van den Bongard, ${ }^{3}$ Sofie A M Gernaat, ${ }^{4}$ Nikolas Lessmann, ${ }^{\circledR} 2$ \\ Margriet G A Sattler, ${ }^{5}$ Arco J Teske, ${ }^{6}$ Joan Penninkhof, ${ }^{5}$ Hanneke Meijer, ${ }^{7}$ \\ Jean-Philippe Pignol, ${ }^{8}$ Helena M Verkooijen, ${ }^{1,9}$ Bragatston study group
}

To cite: Emaus MJ, Išgum I, van Velzen SGM, et al. Bragatston study protocol: a multicentre cohort study on automated quantification of cardiovascular calcifications on radiotherapy planning CT scans for cardiovascular risk prediction in patients with breast cancer. BMJ Open 2019;9:e028752. doi:10.1136/ bmjopen-2018-028752

- Prepublication history for this paper is available online. To view these files, please visit the journal online (http://dx.doi. org/10.1136/bmjopen-2018028752).

Received 21 December 2018 Revised 25 May 2019 Accepted 7 June 2019

D Check for updates

(c) Author(s) (or their employer(s)) 2019. Re-use permitted under CC BY-NC. No commercial re-use. See rights and permissions. Published by BMJ.

For numbered affiliations see end of article.

Correspondence to Professor Dr Helena

M Verkooijen;

h.m.verkooijen@umcutrecht.nl

\section{ABSTRACT}

Introduction Cardiovascular disease (CVD) is an important cause of death in breast cancer survivors.

Some breast cancer treatments including anthracyclines, trastuzumab and radiotherapy can increase the risk of CVD, especially for patients with pre-existing CVD risk factors. Early identification of patients at increased CVD risk may allow switching to less cardiotoxic treatments, active surveillance or treatment of CVD risk factors. One of the strongest independent CVD risk factors is the presence and extent of coronary artery calcifications (CAC). In clinical practice, CAC are generally quantified on ECGtriggered cardiac CT scans. Patients with breast cancer treated with radiotherapy routinely undergo radiotherapy planning CT scans of the chest, and those scans could provide the opportunity to routinely assess CAC before a potentially cardiotoxic treatment. The Bragatston study aims to investigate the association between calcifications in the coronary arteries, aorta and heart valves (hereinafter called 'cardiovascular calcifications') measured automatically on planning CT scans of patients with breast cancer and CVD risk.

Methods and analysis In a first step, we will optimise and validate a deep learning algorithm for automated quantification of cardiovascular calcifications on planning CT scans of patients with breast cancer. Then, in a multicentre cohort study (University Medical Center Utrecht, Utrecht, Erasmus MC Cancer Institute, Rotterdam and Radboudumc, Nijmegen, The Netherlands), the association between cardiovascular calcifications measured on planning CT scans of patients with breast cancer ( $n \approx 16000$ ) and incident (non-)fatal CVD events will be evaluated. To assess the added predictive value of these calcifications over traditional CVD risk factors and treatment characteristics, a case-cohort analysis will be performed among all cohort members diagnosed with a CVD event during follow-up ( $\mathrm{n} \approx 200$ ) and a random sample of the baseline cohort $(n \approx 600)$.
Strengths and limitations of this study

- For each patient, an individual cardiovascular risk score will be automatically calculated on routine radiotherapy planning CT scans.

- Cardiovascular calcifications will be measured using an automated deep learning algorithm in an objective, reproducible and fast manner.

- A case-cohort design will be used to estimate absolute risks, which will facilitate clinical (shared) decision making.

- Outcome data will be obtained through linkage with high-quality national registries.

- Due to the relatively short follow-up, the number of long-term cardiovascular disease events will be limited which may lead to an underestimation of the prognostic value of cardiovascular calcifications.

Ethics and dissemination The Institutional Review Boards of the participating hospitals decided that the Medical Research Involving Human Subjects Act does not apply. Findings will be published in peer-reviewed journals and presented at conferences.

Trial registration number NCT03206333.

\section{INTRODUCTION}

Over the past 25 years, breast cancer mortality rates have declined substantially following improvements in therapy and early detection due to screening. ${ }^{2}$ This, in combination with high breast cancer incidence rates, has resulted in a considerable number of breast cancer survivors. ${ }^{3}$ In 2012, there were 6.2 million women worldwide who had been diagnosed with breast cancer in the previous 5 years and many of them are assumed to die 
of causes unrelated to breast cancer. ${ }^{34}$ This implies a strong need for research on prevention of breast cancer treatment-induced complications, such as cardiovascular diseases (CVDs).

CVD is the leading cause of death in women worldwide accounting for one third of all global female deaths in 2015. Also in patients with breast cancer it is an important cause of mortality. ${ }^{4}$ Colzani $e t a l$ showed that in patients with breast cancer $12 \%$ of all deaths within 10 years after diagnosis were attributed to CVD, and in the subgroup of elderly patients ( $>65$ years) $24 \%$ of deaths were CVD-related. ${ }^{6}$ Radiotherapy and some systemic therapies such as chemotherapy (anthracyclines) and immunotherapy (trastuzumab) can increase the risk of CVD, in particular in patients with pre-existing CVD risk factors. ${ }^{7-13}$ Early and accurate identification of patients at increased risk of CVD, i.e. before breast cancer treatment is administered, is important to reduce the burden of CVD in breast cancer survivors.

One of the strongest independent CVD risk factors is the presence and extent of coronary artery calcifications (CAC) ${ }^{14}$ In clinical practice, CAC are quantified on ECG-synchronised cardiac CT scans without contrast. All patients with breast cancer who receive radiation therapy ( $>60 \%$ of patients with breast cancer ${ }^{15}$ ) routinely undergo a radiotherapy (RT) planning CT scan of the chest. Although these scans do not have the same image quality for the detection of CAC as cardiac scans due to the absence of ECG triggering and lower image resolution, it has been shown that CAC can still be routinely assessed on these scans. ${ }^{16}{ }^{17} \mathrm{CAC}$ was measured using an automated deep learning algorithm which has the advantage of being an objective, reproducible and fast method. One in four patients with breast cancer had some degree of CAC. ${ }^{16}{ }^{17}$ Based on information from RT medical records, $28 \%$ of patients with severe CAC did not have other traditional CVD risk factors. ${ }^{16}$ However, evidence on whether CAC measured on RT planning CT scans is a predictor of CVD risk is still lacking. In addition, the prognostic value of CAC has not yet been investigated in patients with breast cancer.

If patients with breast cancer at increased risk of CVD can be identified, these patients may benefit from less cardiotoxic treatment strategies, for example, adaptation of RT target volumes or technique, chemotherapy dose reduction or switching to less harmful regimes, an intervention on CVD risk factors including lifestyle changes or pharmacoprevention and from close monitoring for early detection of cardiotoxicity during and after breast cancer treatment using imaging techniques or biomarkers. ${ }^{18-30}$ In that way, the burden of CVD among breast cancer survivors could be reduced and lead to a better overall survival rate and improved quality of life.

The Bragatston study aims to investigate the association between CAC measured automatically on RT planning CT scans using a deep learning algorithm and CVD risk among patients with breast cancer. Furthermore, thoracic aorta calcifications (TAC), aortic valve calcifications
(AVC) and mitral valve calcifications (MVC) will also be analysed as they are also associated with CVD risk. ${ }^{31-33}$ In this manuscript, we report the design of the Bragatston study.

\section{METHODS AND ANALYSIS \\ Study aims}

The Bragatston study is divided into three work packages (WP):

- WP 1: this diagnostic package aims to optimise and validate an in-house developed automated deep learning algorithm to measure the presence and extent of CAC, TAC, AVC and MVC (hereinafter called 'cardiovascular calcifications') on RT planning CT scans of patients with breast cancer.

- WP 2: this aetiological package will evaluate the association between cardiovascular calcifications measured automatically on RT planning CT scans and the risk of (non-)fatal CVD events in patients with breast cancer. It will also evaluate if the association is modified by type of (neo-)adjuvant breast cancer treatment.

- WP 3: this prognostic package will assess the added value of cardiovascular calcifications measured automatically on RT planning CT scans over traditional CVD risk factors and breast cancer treatment characteristics to predict (non-)fatal CVD events in patients with breast cancer.

\section{Study design and population}

For WP 1 and 2, the Bragatston study uses a cohort design (figure 1). The cohort will include all patients with non-metastatic primary breast cancer treated with RT at the University Medical Center Utrecht, Utrecht, the Erasmus MC Cancer Institute, Rotterdam and the Radboudumc, Nijmegen $(n \approx 16000)$, the Netherlands. Patients with a prevalent cancer diagnosis will be excluded. From these institutions, RT planning CT scans and clinical data will be collected starting from the time CT RT planning was introduced, which was in 2005 (University Medical Center Utrecht) and 2006 (Erasmus MC Cancer Institute and Radboudumc) until the end of 2016. The RT planning CT scans that will be collected are acquired as part of clinical routine (no contrast enhancement, no ECG triggering, $120 \mathrm{kVp}$, in-plane resolution $0.78-1.37 \mathrm{~mm}, 3.0 \mathrm{~mm}$ slice thickness, $3.0 \mathrm{~mm}$ increment).

For WP 3, a case-cohort study will be conducted. ${ }^{34}$ The case-cohort study will include all cohort members diagnosed with a CVD event during follow-up, called hereafter cases. In addition, a random sample will be selected at baseline from the cohort to serve as control. To increase statistical power, a case-to-control ratio of 1:3 will be applied leading to a random sample of approximately 600 patients. The power gained by including more than three controls to one case is little. 


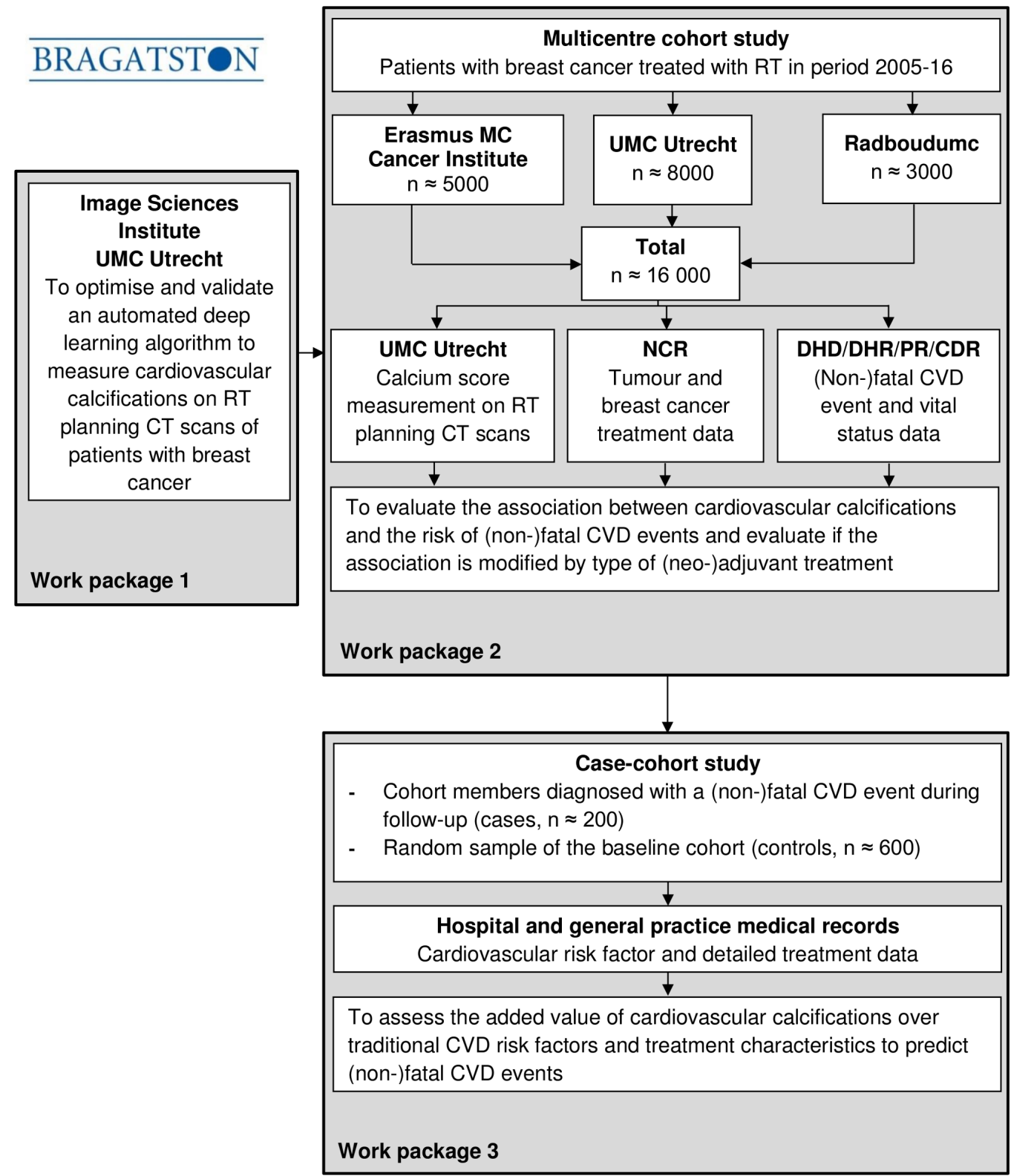

Figure 1 Flow chart of Bragatston study. CDR, National Cause of Death Register; CVD, cardiovascular disease; DHD, Dutch Hospital Data; DHR, Dutch Heart Registration; NCR, Netherlands Cancer Registry; PR, Dutch Population Register; RT, radiotherapy; UMC, University Medical Center.

\section{Data collection procedures}

\section{Automatic calcification quantification}

Cardiovascular calcifications will be measured automatically using a calcium scoring algorithm previously developed in our group. ${ }^{35}$ The algorithm uses two consecutive convolutional neural networks (CNN) to label voxels as calcifications in the coronary arteries (left main coronary artery, left anterior descending artery, left circumflex artery and right coronary artery), as well as calcifications in the thoracic aorta and the aortic and mitral valve (leaflets and annulus). The first CNN is used on a large field of view to enable learning from contextual spatial information. This CNN is able to identify calcified voxels and label them according to their anatomical location. The second CNN uses a smaller field of view and analyses the detailed local texture. This CNN can differentiate the true atherosclerotic calcifications among the candidates detected by the first CNN (figures 2-5).

The algorithm was initially developed to analyse low-dose lung cancer screening CT scans. ${ }^{35}$ Hence, the algorithm has been modified to be able to measure calcifications on RT planning CT scans of patients with breast cancer. This procedure has been described in detail elsewhere. ${ }^{17}$ The first results show that automatic calcification quantification is possible on RT planning CT scans of patients with breast cancer. Reproducibility of automatically versus manually measured calcium scores was high with linearly weighted kappa values $\geq 0.84$ and intraclass correlation coefficients $\geq 0.94 .{ }^{17}$ For the current project, we will further develop the method to ensure its robustness 


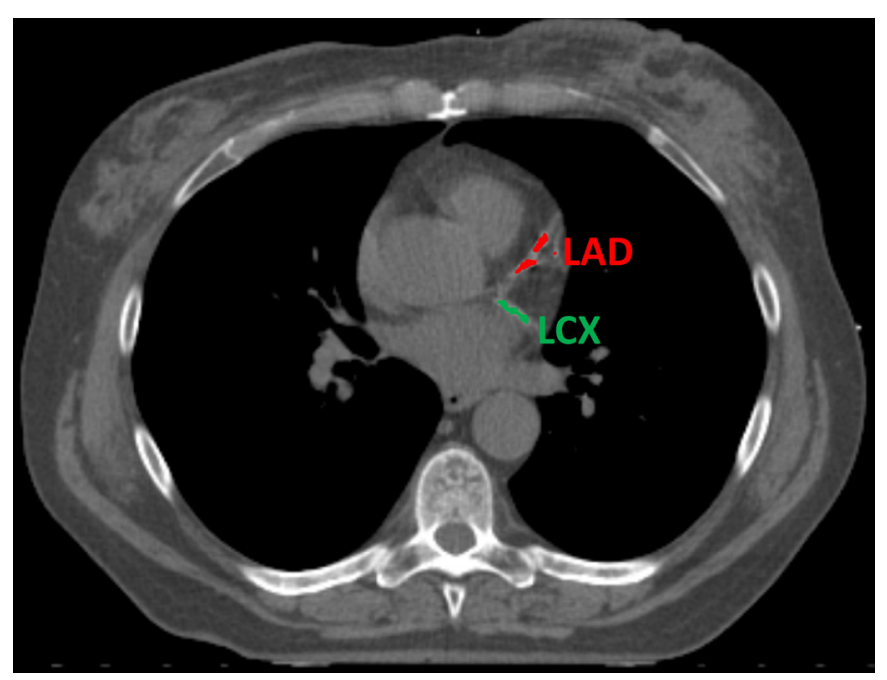

Figure 2 Example of automatic calcification quantification on radiotherapy planning CT scan using our deep learning algorithm. Radiotherapy planning CT scan image showing calcifications in the left anterior descending artery (in red) and left circumflex artery (in green). LAD, left anterior descending artery; LCX, left circumflex artery.

with respect to image acquisition parameters and thereby enable its applicability in multicentre settings.

CAC, TAC, AVC and MVC will be expressed in volume scores (in $\mathrm{mm}^{3}$ ). For routine ECG-gated cardiac CT scans, CAC is expressed in the Agatston score which also takes the calcification density into account. ${ }^{36}$ The CT scans used in this project are ungated and therefore we will report CAC as modified Agatston score. ${ }^{37}$ These modified Agatston scores will be calculated by multiplying the calcification area (in $\mathrm{mm}^{2}$ ) by the density score (1, 130-199 Hounsfield Units (HU); 2, 200-299 HU; 3, 300-399 $\mathrm{HU} ; 4,>399 \mathrm{HU}$ ) of the area (calcification density) and summing the lesion scores, in which a minimal lesion definition of $1.5 \mathrm{~mm}^{3}$ will be maintained to eliminate noise. Based on these scores, patients will be categorised into the Agatston classification consisting of five categories: $0,1-10,11-100,101-400,>400$ Agatston units.

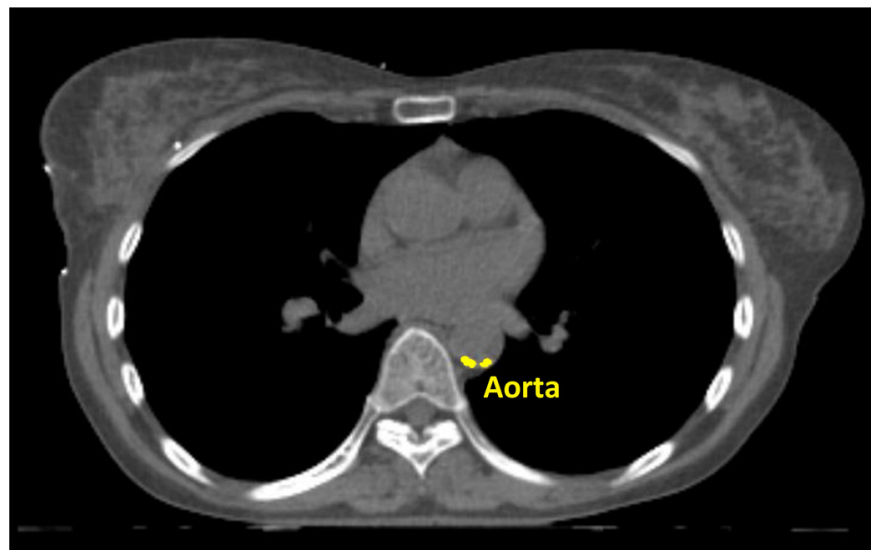

Figure 3 Example of automatic calcification quantification on radiotherapy planning CT scan using our deep learning algorithm. Radiotherapy planning CT scan image showing thoracic aorta calcifications (in yellow).

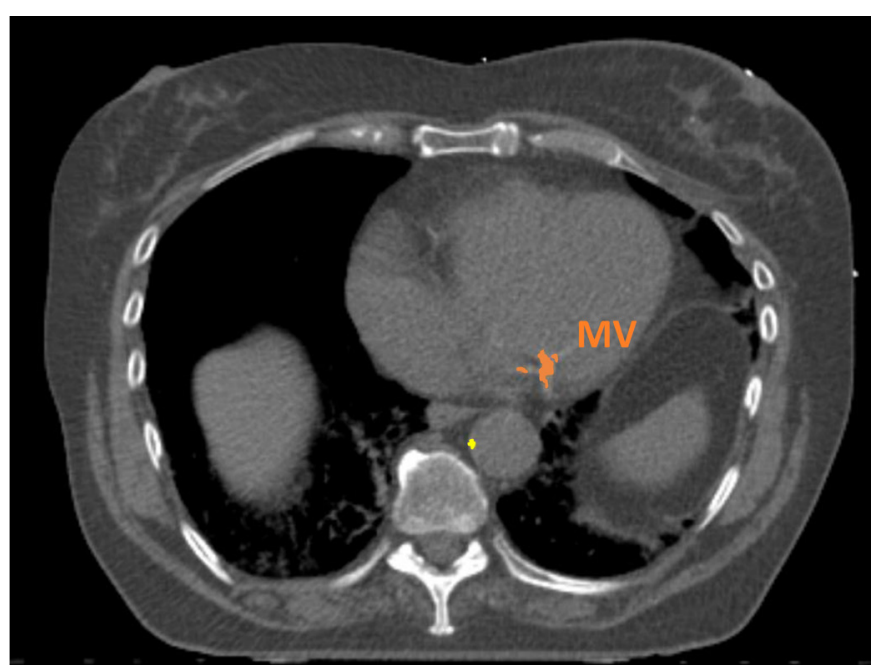

Figure 4 Example of automatic calcification quantification on radiotherapy planning CT scan using our deep learning algorithm. Radiotherapy planning CT scan image showing mitral valve calcifications (in orange) and thoracic aorta calcifications (in yellow). MV, mitral valve.

\section{Reference library of manual calcification quantification}

Reference standard for calcium scoring will be defined by manual calcium scoring. Manual calcium scoring will be done in a subset of planning CT scans randomly selected per hospital (University Medical Center Utrecht: $\mathrm{n}=500$; the Erasmus MC Cancer Institute: $n=300$; Radboudumc: $\mathrm{n}=200$ ). Calcifications in the coronary arteries, the aorta and heart valves will be manually identified and labelled. As is standard procedure, three-dimensional region growing will be used with a threshold of $130 \mathrm{HU}^{36}$ Manual annotation will be performed by observers who will be trained and supervised by a radiologist (PAJ) with $>10$ years of experience in cardiac CT. Subsequently, manually annotated calcifications will be quantified to determine calcium scores. In line with the automatic calcification quantification, CAC, TAC, AVC and MVC will be

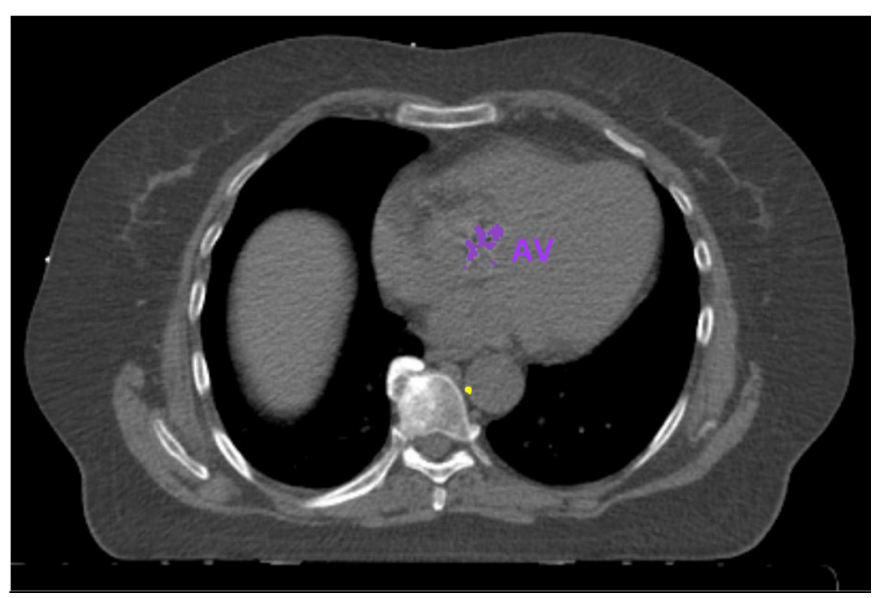

Figure 5 Example of automatic calcification quantification on radiotherapy planning CT scan using our deep learning algorithm. Radiotherapy planning CT scan image showing aortic valve calcifications (in purple) and thoracic aorta calcifications (in yellow). AV, aortic valve. 
expressed in volume scores (in $\mathrm{mm}^{3}$ ). The modified Agatston scores will be calculated as described in the previous section. Those reference annotations will be used to train the algorithm and to evaluate its performance.

\section{Tumour and treatment characteristics and CVD risk factors}

Tumour and breast cancer treatment data will be obtained through linkage with the Netherlands Cancer Registry (NCR) hosted by the Netherlands Comprehensive Cancer Organisation. ${ }^{38}$ Tumour data variables include tumour stage, grade and receptor status and treatment data variables include type of surgery (breast conserving therapy, mastectomy), RT (laterality and radiation fields (if available)), chemotherapy (yes, no), hormonal therapy (yes, no) and immunotherapy (yes, no).

For WP 3, detailed data on breast cancer treatment and traditional CVD risk factors present at breast cancer diagnosis will be extracted from hospital and general practice medical records. The following traditional CVD risk factor data will be collected: age, sex, hypertension, hypercholesterolaemia, diabetes, smoking and body mass index. Regarding hypertension, hypercholesterolaemia, diabetes and smoking, a patient will be scored positive when the risk factor is documented in the hospital medical record or reported by the general practitioner by means of a questionnaire. Local project members of the participating hospitals will perform linkage with the NCR and will collect medical record data.

\section{Assessment of outcome}

The primary outcome is the incidence of (non-) fatal CVD events, which is defined as hospitalisation or death from CVD. Death from CVD will be recorded if it is primary cause of death, meaning this is the disease that leads to death. CVD outcomes will be captured through linkage with Dutch Hospital Data (DHD), the Dutch Heart Registration (DHR), the Dutch Population Register (PR) and the National Cause of Death Register (CDR).

DHD collects nationwide medical and administrative data for all inpatient and day hospital-care in the Netherlands (ie, Hospital Discharge Register). The DHD uses the International Classification of Disease ninth revision (ICD-9). ${ }^{39}$ According to this classification, CVD will be categorised as diseases of the circulatory system (ICD codes 390-459) and will be further subcategorised into the following subcategories: hypertensive disease (401-405), ischaemic heart disease (410-414), pericarditis (420), valvular dysfunction (424), cardiomyopathy (425), arrhythmia (426-427), heart failure (428) and cerebrovascular disease (430-438) and other. Linkage with the DHD will be facilitated by Statistics Netherlands using the record identification number. ${ }^{40}$ This number is based on a combination of date of birth, sex and postal code and is assigned to each resident in the Netherlands.

For a more complete data collection on incident CVD, additional linkage with the DHR will be performed. ${ }^{41}$ The DHR collects data on cardiac interventions (eg, percutaneous coronary intervention) and cardiothoracic surgery (eg, coronary artery bypass surgery, heart valve surgery). Linkage will be performed using a combination of identifiers including date of birth, sex and maiden name.

Data on vital status will be obtained from the Dutch Population Register (PR). Causes of death will be obtained from the CDR maintained by Statistics Netherlands. The register contains information on all primary and secondary causes of death from all deceased persons registered in the Netherlands. Causes of death are classified according to ICD-10. ${ }^{42}$ CVD mortality will be categorised as diseases of the circulatory system (ICD codes I00-I99) and will be further subcategorised into the following subcategories: hypertensive diseases (I10-I13), ischaemic heart diseases (I20-I25), pericarditis (I30-I32), valvular dysfunction (I34-I38), cardiomyopathy (I42), arrhythmia (I44-I49), heart failure (I50) and cerebrovascular diseases (I60-I69) and other. Linkage with the PR and CDR will be provided by Statistics Netherlands. Linkage will be performed by local project members of the participating hospitals. Registries are complete until the end of 2016 (DHD and DHR) or 2017 (CDR).

\section{Power calculation}

The cohort will consist of approximately 16000 patients with breast cancer (University Medical Center Utrecht: $\mathrm{n} \approx 8000$; Erasmus $\mathrm{MC}$ Cancer Institute: $\mathrm{n} \approx 5000$; Radboudumc: $n \approx 3000$ ). A preliminary study was conducted within the prospective breast cancer cohort Utrecht cohort for Multiple BREast cancer intervention studies and Long-term evaLuAtion (UMBRELLA). ${ }^{16} 43$ In total, 561 UMBRELLA patients were included and $24 \%$ of them had CAC (ie, Agatston score $>0$ ) ${ }^{16}$ By including at least 12000 patients with an average follow-up of 4 years, 2880 patients will be expected to have CAC. Assuming $4.5 \%$ risk of CVD events after 4 years of follow-up, 130 CVD events among patients with CAC are expected. ${ }^{44}$ In the 9120 patients without CAC, with a CVD risk of $1.5 \%$ after 4 years, 137 CVD events are expected. Based on the expected number of at least 200 cases of CVD, a maximum of 20 predictor variables can be selected for predicting CVD without risk of overfitting. ${ }^{45}$

\section{Statistical analysis}

For WP 1, reliability and agreement will be assessed between automatically and manually determined calcium scores. Results will be presented for the total sample of 1000 manually and automatically assessed planning CT scans. In addition, results will be stratified by participating hospital. Agreement between continuous calcium scores will be assessed using Bland-Altman plots and between calcium score categories using proportional agreement. To determine reliability, intraclass correlation coefficients will be calculated for continuous calcium scores. Reliability of calcium categories will be evaluated as Cohen's linearly weighted kappa.

For WP 2, (non-)fatal CVD event rates per 1000 person years will be calculated for each calcium score category and plotted using Kaplan-Meier survival curves. Differences 
between categories will be tested with log-rank tests. Cox proportional hazard models will be used to examine the association between calcium scores and (non-)fatal CVD events. Results will be expressed as HRs with their corresponding 95\% CIs. Follow-up time will be the underlying time variable starting from the date of RT planning CT scan and ending at the date of diagnosis of (non-)fatal CVD event or censoring. Censored observations will include non-cardiovascular death, diagnosis of other cancers or end of follow-up, whichever came first. Models will be adjusted for age at RT planning CT scan and calendar year of RT planning CT scan. To assess possible effect modification of the association between cardiovascular calcifications and CVD risk by cardiotoxic chemotherapy, left-sided RT or trastuzumab, stratified analysis will be performed. If evidence for effect modification will be found, models with and without the cross-product term for calcium score and cardiotoxic treatment will be compared using a log-likelihood ratio test. In order to assess the potential effect of competing events precluding the outcome of interest, sensitivity analyses will be conducted comprising cumulative incidence analysis and competing risk survival analysis as described by Fine and Gray. ${ }^{46}$

For WP 3, in univariable cox regression analysis, we will identify which patient characteristics, traditional CVD risk factors or breast cancer treatment characteristics are associated with the risk of CVD events. As proposed by Prentice, a weighted cox regression model will be applied to account for the case-cohort design. ${ }^{34}$ Subsequently, a prediction model will be developed including patient characteristics, traditional CVD risk factors and treatment characteristics. In a second prediction model, calcium scores will be added and the incremental value of calcium scores in CVD risk prediction will be evaluated by comparing discrimination (c-statistics) and reclassification (net reclassification index). To take into account the potential effect of missing data, a sensitivity analysis will be conducted imputing missing values of traditional CVD risk factor and breast cancer treatment variables using multiple imputation. $^{47}$

\section{Patient and public involvement}

We will conduct a survey among 100 UMBRELLA patients to explore their preferences regarding disclosure of calcium scores and corresponding CVD risk. Themes that will be included in the questionnaire are patient's knowledge about CVD risk following a breast cancer diagnosis, the patient's wish to be informed about CVD risk and preferences on way of disclosure of CVD risk. The survey will be developed in collaboration with the Dutch Patient Advocacy Group, a joint initiative from the Dutch Breast Cancer Research Group and the Dutch Breast Cancer Association. ${ }^{48}{ }^{49}$ We will inform patients with breast cancer about the results of this project by means of newsletters and presentations at patient conferences, for example, at the annual UMBRELLA patient conference.

\section{Timeline}

Data collection started in January 2017 and we expect to complete data collection in December 2019. The estimated end date of the study is March 2020.

\section{Ethics and dissemination}

The Institutional Review Boards decided that the Medical Research Involving Human Subjects Act does not apply to the study. The requirement for informed consent was waived in accordance with the Code of Conduct for Medical Research developed by the Federation of Medical Scientific Societies. ${ }^{50}$ All data, with the exception of data provided by Statistics Netherlands, will be stored centrally at the University Medical Center Utrecht. This dataset will be sent to Statistics Netherlands for additional linkage. Analyses will be performed in a secure environment of Statistics Netherlands. The dataset will be anonymised by Statistics Netherlands. The results of the Bragatston study will be published in international peer-reviewed journals and presented at scientific conferences.

\section{DISCUSSION}

The Dutch Bragatston study has been set up to optimise and validate an automated deep learning algorithm for the identification of patients with breast cancer at high risk of CVD based on the presence of cardiovascular calcifications on RT planning CT. Most breast cancer treatment guidelines and survival prediction tools mainly focus on tumour characteristics while other patient characteristics are hardly taken into account. In the era of personalised medicine, given the high burden of CVD in patients with breast cancer it is critical to incorporate patient CVD risk factors in treatment decisions to find an optimal balance between cancer control and cardiotoxicity. Automated measurement of cardiovascular calcifications on RT planning CT scans may be an elegant solution, because RT planning CT scans are readily available imaging data and therefore there is no additional (radiation exposure) harm to patients and only a minimal financial burden to society when calcium scores are measured on these scans.

If we find that cardiovascular calcifications measured on RT planning CT scans are predictors of CVD risk, the next step will be to investigate how to act on this information and how to disclose this information to the patient. The dilemma of disclosing calcium scores and corresponding CVD risk lies in the fact that there is no evidence yet regarding effective risk reducing interventions. Thus far, no randomised trials have been conducted on the effectiveness of calcium score-based treatment strategies with CVD morbidity and mortality reduction as outcome measure. ${ }^{51}$ The Risk Or Benefit IN Screening for CArdiovascular diseases (ROBINSCA) trial is the first ongoing randomised controlled trial investigating the value of CAC imaging followed by preventive treatment in reducing coronary heart disease-related mortality and morbidity. ${ }^{52}$ In the intervention arm, participants with a CAC Agatston score $>100$ will be treated with 
statins and ACE inhibitors, independent of their blood cholesterol level and blood pressure value. The results of the ROBINSCA trial might provide important insights potentially relevant for patients with breast cancer with moderate or high CAC score.

The importance of our study lies in the possibility to introduce targeted preventive interventions to reduce treatment-related CVD. Those include minimisation of the mean RT heart dose, for example, by application of volumetric modulated arc therapy instead of the standard three-dimensional conformal radiation therapy. ${ }^{53}$ Furthermore, chemotherapy-induced cardiotoxicity may be reduced by switching to less harmful regimens, for example, an anthracycline-free regime consisting of docetaxel, carboplatin and trastuzumab has been described for human epidermal growth factor receptor 2-positive breast cancer as a more heart-friendly alternative to the standard regimen. ${ }^{19} 2127$ Another strategy is to screen for and treat modifiable cardiovascular risk factors like high blood pressure, diabetes mellitus and high cholesterol levels. ${ }^{18-20}$ Additionally, increased awareness needs to be generated among physicians to identify and refer patients with breast cancer at high risk for CVD to a cardio-oncologist. Cardio-oncology is a new upcoming discipline focused on cardiovascular care for patients with cancer which comprises CVD risk stratification, close monitoring during and after cancer treatment by means of imaging techniques or circulating biomarkers and management of a possible CVD event. ${ }^{18} 2027$

In conclusion, over the last two decades, advances in breast cancer treatments has led to improved survival rates. However, these treatments can increase the risk of CVD. To optimise the individual benefit and risk evaluation of treatment options, we propose to evaluate the inclusion of information on patient CVD risk. Automated measurement of cardiovascular calcifications on routinely obtained RT planning CT scans may be an inexpensive, fast and accurate solution. The Bragatston study will determine the correlation between those RT planning CT detected cardiovascular calcifications and the occurrence of CVD events.

\section{Author affiliations}

${ }^{1}$ Imaging Division, University Medical Center Utrecht, Utrecht, The Netherlands

${ }^{2}$ Image Sciences Institute, University Medical Center Utrecht, Utrecht, The

Netherlands

${ }^{3}$ Department of Radiation Oncology, University Medical Center Utrecht, Utrecht, The Netherlands

${ }^{4}$ Julius Center for Health Sciences and Primary Care, University Medical Center Utrecht, Utrecht, The Netherlands

${ }^{5}$ Department of Radiation Oncology, Erasmus MC Cancer Institute, Rotterdam, The Netherlands

${ }^{6}$ Department of Cardiology, University Medical Center Utrecht, Utrecht, The Netherlands

${ }^{7}$ Department of Radiation Oncology, Radboudumc, Nijmegen, The Netherlands ${ }^{8}$ Department of Radiation Oncology, Dalhousie University, Halifax, Nova Scotia, Canada

${ }^{9}$ Utrecht University, Utrecht, The Netherlands

Acknowledgements The authors gratefully acknowledge the Dutch Cancer Society for the financial support. They also acknowledge the members of the
Bragatston study group who serve as scientific advisors: Dr Rhodé M Bijlsma, Department of Medical Oncology, University Medical Center Utrecht, Utrecht, the Netherlands; Dr Erwin LA Blezer, Imaging Division, University Medical Center Utrecht, Utrecht, the Netherlands; Professor Dr Michiel L Bots, Julius Center for Health Sciences and Primary Care, University Medical Center Utrecht, Utrecht, the Netherlands and Utrecht University, Utrecht, the Netherlands; Drs Henrike J Bretveld, Department of Research, Netherlands Comprehensive Cancer Organisation (IKNL), Utrecht, the Netherlands; Dr Maartje J Hooning, Department of Medical Oncology, Erasmus MC Cancer Institute, Rotterdam, the Netherlands; Professor Dr Luca Incrocci, Department of Radiation Oncology, Erasmus MC Cancer Institute, Rotterdam, the Netherlands; Professor Dr Pim A de Jong, Department of Radiology, University Medical Center Utrecht, Utrecht, the Netherlands and Utrecht University, Utrecht, the Netherlands; Professor Dr Tim Leiner, Department of Radiology, University Medical Center Utrecht, Utrecht, the Netherlands and Utrecht University, Utrecht, the Netherlands; Dr Julia J van Tol-Geerdink, Department of Radiation Oncology, Radboudumc, Nijmegen, the Netherlands; Dr llonca Vaartjes, Julius Center for Health Sciences and Primary Care, University Medical Center Utrecht, Utrecht, the Netherlands; Dr Wouter B Veldhuis, Department of Radiology, University Medical Center Utrecht, Utrecht, the Netherlands; Dr Janneke Verloop, Department of Research, Netherlands Comprehensive Cancer Organisation (IKNL), Utrecht, the Netherlands; Professor Dr Max A Viergever, Image Sciences Institute, University Medical Center Utrecht, Utrecht, the Netherlands and Utrecht University, Utrecht, the Netherlands; Professor Dr Frank LJ Visseren, Department of Vascular Medicine, University Medical Center Utrecht, Utrecht, the Netherlands and Utrecht University, Utrecht, the Netherlands; Dr Hester Wessels, Department of Corporate Communications, University Medical Center Utrecht, Utrecht, the Netherlands.

Collaborators Bragatston study group: R M Bijlsma; E L A Blezer; M L Bots; $\mathrm{H} \mathrm{J}$ Bretveld; M J Hooning; L Incrocci; P A de Jong; T Leiner; J J van Tol-Geerdink; I Vaartjes; W B Veldhuis; J Verloop; M A Viergever; F L J Visseren; H Wessels.

Contributors MJE, II, SGMV, HJGDB, SAMG, NL, MGAS, AJT, JP, HM, J-PP, HMV set up the study and protocols. MJE, II, SGMV, SAMG and HMV drafted the manuscript. All authors read and approved the final manuscript.

Funding The work is supported by the Dutch Cancer Society (grant number UU 2015-7947).

Competing interests II: disclosures not related to the present article: II received research grants from 1) PIE Medical Imaging BV; 2) the Netherlands Organization for Health Research and Development (ZonMw) with participation of PIE Medical Imaging BV; 3) the Dutch Technology Foundation (STW) within Deep Learning for Medical Image Analysis (DLMedIA) with participation of PIE Medical Imaging BV and Philips Healthcare; 4) Dutch Technology Foundation (STW) with participation of PIE Medical Imaging BV and 3mensio Medical Imaging. In addition, II has a patent, US Patent Application Number 15/933854, with royalties paid and she is scientific founder and a shareholder of Quantib-U BV. JP: disclosures not related to the present article: JP received grants from 1) Accuray, Sunnyvale, California, USA; 2) Elekta AB,Stockholm, Sweden.

\section{Patient consent for publication Not required.}

Ethics approval The study protocol has been reviewed by the Institutional Review Boards of the University Medical Center Utrecht (reference number: 16- 721 /C), Erasmus MC Cancer Institute Rotterdam (MEC-2017-1125) and Radboudumc (2017-3847).

Provenance and peer review Not commissioned; externally peer reviewed.

Open access This is an open access article distributed in accordance with the Creative Commons Attribution Non Commercial (CC BY-NC 4.0) license, which permits others to distribute, remix, adapt, build upon this work non-commercially, and license their derivative works on different terms, provided the original work is properly cited, appropriate credit is given, any changes made indicated, and the use is non-commercial. See: http://creativecommons.org/licenses/by-nc/4.0/.

\section{REFERENCES}

1. Berry DA, Cronin KA, Plevritis SK, et al. Effect of screening and adjuvant therapy on mortality from breast cancer. $N$ Engl J Med 2005;353:1784-92

2. Plevritis SK, Munoz D, Kurian AW, et al. Association of Screening and Treatment With Breast Cancer Mortality by Molecular Subtype in US Women, 2000-2012. JAMA 2018;319:154-64.

3. Ferlay J, Soerjomataram I, Ervik M, et al. GLOBOCAN 2012 v1.0, Cancer Incidence and Mortality Worldwide: IARC CancerBase No. 
11 [Internet]. Lyon, France: International Agency for Research on Cancer. 2013 http://globocan.iarc.fr (accessed 17 May 2018).

4. Gernaat SAM, Ho PJ, Rijnberg N, et al. Risk of death from cardiovascular disease following breast cancer: a systematic review. Breast Cancer Res Treat 2017;164:537-55.

5. World Health Organization. Global health estimates 2015: deaths by cause, age and sex, by country and by region, 2000-2015. http:// www.who.int/healthinfo/global_burden_disease/estimates/en/index1. html (accessed 17 May 2018).

6. Colzani E, Liljegren A, Johansson AL, et al. Prognosis of patients with breast cancer: causes of death and effects of time since diagnosis, age, and tumor characteristics. $J$ Clin Oncol 2011;29:4014-21.

7. Darby SC, Ewertz M, McGale P, et al. Risk of ischemic heart disease in women after radiotherapy for breast cancer. $N$ Engl $J$ Med 2013;368:987-98.

8. Hooning MJ, Botma A, Aleman BM, et al. Long-term risk of cardiovascular disease in 10-year survivors of breast cancer. $J$ Natl Cancer Inst 2007;99:365-75.

9. Moslehi $\mathrm{J}$. The cardiovascular perils of cancer survivorship. N Engl J Med 2013;368:1055-6.

10. Harris EE. Cardiac mortality and morbidity after breast cancer treatment. Cancer Control 2008;15:120-9.

11. Accordino MK, Neugut Al, Hershman DL. Cardiac effects of anticancer therapy in the elderly. J Clin Oncol 2014;32:2654-61.

12. Brower V. Cardiotoxicity debated for anthracyclines and trastuzumab in breast cancer. J Natl Cancer Inst 2013;105:835-6.

13. Boekel NB, Jacobse JN, Schaapveld M, et al. Cardiovascular disease incidence after internal mammary chain irradiation and anthracycline-based chemotherapy for breast cancer. Br J Cancer 2018;119:408-18.

14. Yeboah J, McClelland RL, Polonsky TS, et al. Comparison of novel risk markers for improvement in cardiovascular risk assessment in intermediate-risk individuals. JAMA 2012;308:788-95.

15. Mittmann N, Seung SJ, Liu N, et al. Population-based utilization of radiation therapy by a Canadian breast cancer cohort. Curr Oncol 2014;21:715-7.

16. Gernaat SA, Išgum I, de Vos $\mathrm{BD}$, et al. Automatic Coronary Artery Calcium Scoring on Radiotherapy Planning CT Scans of Breast Cancer Patients: Reproducibility and Association with Traditional Cardiovascular Risk Factors. PLoS One 2016;11:e0167925.

17. Gernaat SAM, van Velzen SGM, Koh V, et al. Automatic quantification of calcifications in the coronary arteries and thoracic aorta on radiotherapy planning CT scans of Western and Asian breast cancer patients. Radiother Oncol 2018;127:487-92.

18. Zamorano JL, Lancellotti P, Rodriguez Munoz D, et al. ESC Position Paper on cancer treatments and cardiovascular toxicity developed under the auspices of the ESC Committee for Practice Guidelines: The Task Force for cancer treatments and cardiovascular toxicity of the European Society of Cardiology (ESC). Eur Heart $J$ 2016;2016:2768-801.

19. Eschenhagen T, Force T, Ewer MS, et al. Cardiovascular side effects of cancer therapies: a position statement from the Heart Failure Association of the European Society of Cardiology. Eur J Heart Fail 2011;13:1-10.

20. Armenian SH, Lacchetti C, Barac A, et al. Prevention and Monitoring of Cardiac Dysfunction in Survivors of Adult Cancers: American Society of Clinical Oncology Clinical Practice Guideline. J Clin Oncol 2017;35:893-911.

21. Levis BE, Binkley PF, Shapiro CL. Cardiotoxic effects of anthracycline-based therapy: what is the evidence and what are the potential harms? Lancet Oncol 2017;18:e445-56.

22. Ades F, Zardavas D, Pinto AC, et al. Cardiotoxicity of systemic agents used in breast cancer. Breast 2014;23:317-28.

23. Witteles RM, Bosch X. Myocardial Protection During Cardiotoxic Chemotherapy. Circulation 2015;132:1835-45.

24. Kalam K, Marwick TH. Role of cardioprotective therapy for prevention of cardiotoxicity with chemotherapy: a systematic review and meta-analysis. Eur J Cancer 2013;49:2900-9.

25. Bruzzaniti V, Abate A, Pinnarò P, et al. Dosimetric and clinical advantages of deep inspiration breath-hold (DIBH) during radiotherapy of breast cancer. J Exp Clin Cancer Res 2013;32:88

26. Curigliano G, Cardinale D, Suter T, et al. Cardiovascular toxicity induced by chemotherapy, targeted agents and radiotherapy: ESMO Clinical Practice Guidelines. Ann Oncol 2012;23(Suppl 7):vii155-66.

27. Comprehensive Cancer Centre the Netherlands (IKNL). Nationwide Breast Cancer Guideline https://www.oncoline.nl/borstkanker (accessed 17 May 2018).
28. Dang CT, Yu AF, Jones LW, et al. Cardiac Surveillance Guidelines for Trastuzumab-Containing Therapy in Early-Stage Breast Cancer: Getting to the Heart of the Matter. J Clin Oncol 2016;34:1030-3.

29. Curigliano G, Cardinale D, Dent S, et al. Cardiotoxicity of anticancer treatments: Epidemiology, detection, and management. CA Cancer $J$ Clin 2016;66:309-25.

30. Correa C, Harris EE, Leonardi MC, et al. Accelerated Partial Breast Irradiation: Executive summary for the update of an ASTRO Evidence-Based Consensus Statement. Pract Radiat Oncol 2017:7:73-9.

31. Tison GH, Guo M, Blaha MJ, et al. Multisite extracoronary calcification indicates increased risk of coronary heart disease and all-cause mortality: The Multi-Ethnic Study of Atherosclerosis. J Cardiovasc Comput Tomogr 2015;9:406-14.

32. Jacobs PC, Prokop M, van der Graaf $\mathrm{Y}$, et al. Comparing coronary artery calcium and thoracic aorta calcium for prediction of allcause mortality and cardiovascular events on low-dose non-gated computed tomography in a high-risk population of heavy smokers. Atherosclerosis 2010;209:455-62.

33. Willemink MJ, Takx RA, Išgum I, et al. Prognostic value of heart valve calcifications for cardiovascular events in a lung cancer screening population. Int J Cardiovasc Imaging 2015;31:1243-9.

34. Prentice RL. A case-cohort design for epidemiologic cohort studies and disease prevention trials. Biometrika 1986;73:1-11.

35. Lessmann N, van Ginneken B, Zreik M, et al. Automatic Calcium Scoring in Low-Dose Chest CT Using Deep Neural Networks With Dilated Convolutions. IEEE Trans Med Imaging 2018;37:615-25.

36. Agatston AS, Janowitz WR, Hildner FJ, et al. Quantification of coronary artery calcium using ultrafast computed tomography. J Am Coll Cardiol 1990;15:827-32.

37. Chiles C, Duan F, Gladish GW, et al. Association of Coronary Artery Calcification and Mortality in the National Lung Screening Trial: A Comparison of Three Scoring Methods. Radiology 2015;276:82-90.

38. Netherlands Comprehensive Cancer Organisation. https://www.iknl. nl (accessed 17 May 2018).

39. World Health Organization. Manual of the International Statistical Classification of Diseases Injuries and Causes of Death. Ninth Revision, vol 1. Geneva: World Health Organization, 1977.

40. Statistics Netherlands. https://www.cbs.nl/en-gb (accessed 17 May 2018).

41. Dutch Heart Registration. https://www.nederlandsehartregistratie.nl (accessed 17 May 2018).

42. World Health Organization. International Statistical Classification of Diseases, Injuries and Related Health Problems. Tenth Revision. Geneva: World Health Organization, 1992.

43. Young-Afat DA, van Gils $\mathrm{CH}$, van den Bongard $\mathrm{H}$, et al. The Utrecht cohort for Multiple BREast cancer intervention studies and Longterm evaLuAtion (UMBRELLA): objectives, design, and baseline results. Breast Cancer Res Treat 2017;164:445-50.

44. Blaha MJ, Budoff MJ, DeFilippis AP, et al. Associations between C-reactive protein, coronary artery calcium, and cardiovascular events: implications for the JUPITER population from MESA, a population-based cohort study. Lancet 2011;378:684-92.

45. Peduzzi P, Concato J, Feinstein AR, et al. Importance of events per independent variable in proportional hazards regression analysis. II. Accuracy and precision of regression estimates. J Clin Epidemiol 1995;48:1503-10.

46. Fine JP, Gray RJ. A Proportional Hazards Model for the Subdistribution of a Competing Risk. J Am Stat Assoc 1999;94:496-509.

47. Rubin DB. Multiple Imputation for Nonresponse in Surveys. New York: J. Wiley \& Sons, 1987.

48. Dutch Breast Cancer Research Group (BOOG). https://www. boogstudycenter.nl (accessed 20 Dec 2018).

49. Dutch Breast Cancer Association (BVN). https://borstkanker.nl (accessed 20 Dec 2018)

50. Federation of Medical Scientific Societies. Code of conduct. https:// www.federa.org/sites/default/files/bijlagen/coreon/gedragscode_ gezondheidsonderzoek.pdf (accessed 17 May 2018).

51. Greenland P, Alpert JS, Beller GA, et al. ACCF/AHA guideline for assessment of cardiovascular risk in asymptomatic adults: a report of the American College of Cardiology Foundation/American Heart Association Task Force on Practice Guidelines. J Am Coll Cardiol 2010;2010:e50-103.

52. Vonder M, van der Aalst CM, Vliegenthart R, et al. Coronary Artery Calcium Imaging in the ROBINSCA Trial: Rationale, Design, and Technical Background. Acad Radiol 2018;25:118-28.

53. Taylor CW, Kirby AM. Cardiac Side-effects From Breast Cancer Radiotherapy. Clin Oncol 2015;27:621-9. 